Marqués Ibáñez, Ana.

Profesora Ayudante doctor, Universidad de La Laguna, Departamento de Bellas Artes.

\title{
Proyectos Artísticos interdisciplinares en Educación artística.
}

\author{
TIPO DE TRABAJO
}

Comunicación.

PALABRAS CLAVE

Arte contemporáneo, interdisciplinar, Artography, investigación, educación artística.

KEY WORDS

Contemporary art, interdisciplinary, Artography, research, art education.

\section{RESUMEN}

Los modelos de proyectos interdisciplinares actuales en educación artística y cultura visual suponen el diseño de una práctica educativa específica basada en arte contemporáneo.

En esta comunicación se exponen proyectos artísticos que están desarrollados bajo este prima conceptual, así como ejemplos de alumnos que han realizado trabajos creativos en relación al arte como modo de expresión con la metodología descrita como Artography por Rita Irwin. Así hablaremos desde un plano teórico y práctico sobre diversos conceptos aplicados en propuestas concretas que trabajan desde la Educación artística, la investigación y el arte contemporáneo como punto de partida.

El arte contemporáneo se ha enriquecido desde otras disciplinas artísticas como: video experimental, instalaciones, fotografía, etc; este concepto supone una ampliación en el desarrollo de la obra artística en su proceso de creación y evolución.

Se pretende distinguir entre pluridisciplinariedad e interdisciplinariedad, el primer término se refiere al estudio del objeto de una sola disciplina por medio de varias a la vez, por ejemplo, se puede estudiar en la obra Monument Arabe, 1985 Christian Boltansky en relación a la fotografía, instalación, escultura, filosofía, historia, la memoria, el cine, etc. La interdisciplinariedad se relaciona con la transferencia de métodos de una disciplina a otra. La interdisciplinariedad pluridisciplinariedad, y la transdisciplinariedad son nociones conceptuales de una misma idea: el conocimiento integrado.

\section{ABSTRACT}

Current models of interdisciplinary projects in art education and visual culture involve the design of a specific educational experience based on contemporary art.

This paper presents a number of art projects conducted under this conceptual approach. In addition, we put forward examples of creative projects carried out by students who employ art as a form of expression, using a methodology described by Rita Irwin as Artography. As a starting point, we adopt a theoretical and practical approach to consider a number of different concepts applied in specific projects to teach research and contemporary art in Art Education. 
Contemporary art has been enriched by other artistic disciplines, such as experimental video, installations, photography, etc., which has broadened the scope for creating and developing works of art.

We intend to make a distinction between pluridisciplinarity and interdisciplinarity. The first term refers to taking an object from one discipline and studying it simultaneously from several disciplines. For example, Monument Arabe, 1985 by Christian Boltanski can be studied in terms of photography, installation, sculpture, philosophy, history, memory, cinema, etc. Interdisciplinarity regards the transfer of methods from one discipline to another. Interdisciplinarity, pluridisciplinarity, and transdisciplinarity are concepts of the same idea: integrated knowledge.

\section{CONTENIDO}

\section{MANIFESTACIONES ARTISTICAS DE INTERDISCIPLINARIEDAD EN EL ARTE}

Interdisciplinar se refiere a lo relativo a más de un área de conocimiento, ya sea un resultado o una metodología. Referirse a interdisciplinar, es un resultado, que une diversas disciplinas y que se relaciona con ellas. Aunque podrían emplearse otros términos, como: multidisciplinar, pluridisciplinar, transmedia, multimedia, transdisciplinar, que no son sinónimos; pero se refieren a conceptos que implican transferencia de metodologías especialidades conocimientos etc.

Lo importante no es el término sino su significado. El concepto postmoderno performance tiene un origen interdisciplinar y el concepto procede de diversas disciplinas.

Interdisciplinar es cuando en la creación se usan disciplinas de distintas especialidades. Se expone la obra de artistas que utilizan esta metodología como: Oskar Schlemmer, John Cage y Merce Cunnigham.

\subsection{OSKAR SCHLEMMER - BALLET TRIADICO}

La principal coreografía de Schlemmer fue Ballet Triádico (1916) realizado antes de su ingreso en la Bauhaus. Su aportación son los innovadores diseños del vestuario y la coreografía. Con tendencia a la abstracción, la exactitud matemática y el rigor de la línea; tanto en los trajes, como en la utilización del espacio. El vestuario se ejecutó en telas y materiales plásticos pesados esto generó problemas de movilidad en los bailarines, que se resolvió de forma creativa adaptando la música y los movimientos.

En 1922 dirige el taller teatral y lo transforma en un referente de la Bauhaus con su obra: Danzas de la Bauhaus. Entre 1922-1925 es maestro en los talleres de escultura y talla, el trabajo de los mismos se dedica a la ejecución de la escenografía y vestuario para el taller de teatro: máscaras, marionetas, trajes, etc. Esto dará continuación a los diseños escenográficos de los futuristas, con experimentaciones cromáticas de efectos lumínicos: ballets de luces, danzas mecánicas o de marionetas.

Es el artista más multidisciplinar de la Bauhaus capaz de fusionar su trabajo como creador con su práctica docente. Muy polifacético, aunque se inicia como pintor, desarrolla una producción artística que incluye: escultura, teatro, danza, dibujo o escenografía.

1.2. JOHN CAGE (Los Ángeles, 1912 - NY, 1992)

Artista conceptual de gran impacto en la modernidad y posmodernidad interesado en la innovación, elementos que compartió con sus contemporáneos en obras como: White Paintings de Rauschenberg (1951) y Box with the Sound of its Own Making de Robert Morris (1961) hasta el mediascape de Exploding Plastic Inevitable de Warhol, estos trabajos buscan nuevos modos de expresión artística multidisciplinar. 
A pesar de que sus primeras obras son aumentos de técnicas dodecafónicas, su música interesó más que por su composición por el modo de producir sonidos, por lo que Cage no se centró solo en la creación musical como producto final, sino en la fase de creación. Entre sus primeras obras la First Construction (in Metal) (1939) amplió los espacios expresivos de la música de percusión incorporando nuevos instrumentos como su "piano preparado", que seguia el ballet "Baccanale" de Syvilla Fort. Utilizo un piano y lo distorsionó incluyendo entre las cuerdas: gomas, tornillos, tuercas. Se hizo célebre y esta técnica se usa en instrumentos actuales. El water-gong es otro instrumento que se creó para un ballet náutico de la Escuela de Educación Física (California).

Como artista interdisciplinar su mejor época fue en los 50, cuando colabora con la compañía de danza Merce Cuninngham y el Black Mountain College, donde presento su Theater piece No1 (1952), considerado el primer happening.

\subsection{MERCE CUNNINGHAM (Washington, 1919 - N.Y, 2009)}

Bailarín y coreógrafo de la compañía de Martha Graham, investigó las posibilidades de la tecnología para la danza contemporánea. Utilizo vídeo, procesó las coreografías con ordenadores, y es junto a John Cage, primer coreógrafo que muestra una escena multimedia interactiva, en Variation $V$ (1965), los bailarines, junto con proyecciones en el escenario crean una representación interactiva.

En 1999 investiga la captura de movimientos para la obra Biped, una escenografía proyectada en planos frontales, posterior y anterior, así como una coreografía virtual. En su grupo de trabajo incluye ingenieros, que utilizan el software LiveForms para construir bailarines virtuales que interactúan con los reales. Otras obras del artista unen tecnología y danza como: EyeSpace (2006); en la que se distribuyen Ipods a los asistentes para que cada uno escuche su propia versión de la musica. La obra, Interscape (2002) con Rauschenberg, que diseño escenografía y vestuario.

Loops (2001-11) de Merce Cunningham y OpenEnded Group es un espectáculo con sensores de movimiento instalados en las manos del bailarín. Es una danza - performance que utiliza las motion captures, grabadas con el bailarín desde diversos ángulos; que origina una representación en tres dimensiones de los movimientos de sus manos. Incluye en la coreografía, un sistema de sensores para captar el movimiento de sus dedos y transcribirlo a través de un código. El audio registra la voz de Cunningham leyendo un diario, con sonidos de piano, que al ser manipulados originan instrumentos que existen digitalmente. Estos sonidos componen la música de Loops Preservation. La aportación de esta obra interdisciplinar tiene un valor social, no solo como investigación en el campo digital, informático, coreográfico, etc; sino para que se creen nuevos proyectos y la obra se reinvente.

\section{INSTITUCIONES ACADEMICAS INTERDISCIPLINARES: BLACK MOUNTAIN COLLEGE}

El Black Mountain College es una institución académica que originó una generación de artistas, con un trabajo interdisciplinar entre 1933 y 1957 en el que colaboraron Cage y Cunningham. Muchos creadores de la vanguardia americana pasaron por la institución y valoran positivamente su metodología que amplía la capacidad de percepción y asociación y permite innovar nuevos esquemas de creación.

El trabajo interdisciplinar incrementa la capacidad perceptiva y asociativa de los autores y crea mediante procedimientos y formas diversas nuevos esquemas mentales. Los procesos creativos son similares en la totalidad de las disciplinas ya sean poetas, músicos y coreógrafos pueden trabajar buscando ritmos, formas y composición; y los procedimientos son transferibles, porque su objetivo común que es la creación artística.

\section{CENTROS Y MEDIALABAS INTERDISCIPLINARES}

Una línea de investigación en la creación artística que se desarrolla con la utilización de Nuevas Tecnologías de la Información y la Comunicación, son los medialabs, en los que hay una interacción entre una estructura organizativa y 
una red de participantes. Se trata de reflexionar sobre los patrones de cooperación transversal e interdisciplinar y de las acciones que producen arte en estos laboratorios, considerados como un modelo de educación artística emergente, en el que participan varias áreas cognitivas. Se analizan no solo a laboratorios de nuevas tecnologías de la información y la comunicación, sino también laboratorios experimentales.

\subsection{PUBLIC ART LABORATORY, PAL}

Laboratorio de arte fundado en 2002 en Berlín, para el desarrollo, intercambio e investigación de conceptos artísticos y culturales relacionados con las nuevas tecnologías y el espacio urbano, para la construcción de una comunidad creativa a través de redes de proyectos de arte en espacios públicos. Buscan realizar proyectos artísticos trabajando con comunidades para intervenir en su entorno.

El Public Art Laboratory, tiene dos áreas de trabajo: Los Proyectos y la Cultura Digital. En los Proyectos, existen tres tipos de propuestas: Esculturas Urbanas, realizadas con luces en el espacio urbano. Arte Público, estudios Móviles y museos Móviles. En la Cultura Digital los: Media Art Show, Mobicases y Zensors.

Algunos de sus proyectos son: Estudios Móviles (2006), proyecto piloto de red internacional de un laboratorio móvil, una producción de artistas, músicos y programadores culturales. Estos estudios viajaron como una plataforma multimedia nómada por Europa.

\subsection{LABORAL}

Es el Centro de Arte y Creación Industrial de Gijón, fundada en 2007 sus objetivos son: la promoción y difusión del arte y la creación industrial, el objetivo de LABoral es transformar los descubrimientos tecnológicos de productos en un bien cultural. El centro no colecciona obras, sino que trata de incentivar y descubrir nuevos talentos artísticos y de innovación industrial, fomentar el conocimiento de las nuevas corrientes artísticas y científicas, proyectando productos de innovación industrial y promoviéndolos en circuitos artísticos.

En 2010 inició propuestas para la conservación del patrimonio cultural de los nuevos formatos en archivos, mediatecas y centros de documentación, el cómo conservar, documentar y poner en uso de forma eficiente los fondos y proyectos de arte. Actividad que se complementa con La apertura de la Mediateca- Archivo, espacio especializado en la consulta, registro, y divulgación del arte actual y sus industrias culturales experimentales. El centro promueve el trabajo en equipos multidisciplinares.

\subsection{FUTURELAB, ARS ELECTRONICA CENTER}

Ubicado en Linz (Austria), este centro busca la interacción entre arte, tecnología y sociedad, las actividades se realizan en equipos interdisciplinares. En el laboratorio, participan 50 artistas e investigadores que trabajan en la concepción, planificación y realización de proyectos conjuntos. Son artistas, informáticos, científicos, físicos, expertos de los medios de comunicación y diseñadores de productos, arquitectos, programadores de juegos, ingenieros de telemática, sociólogos, historiadores del arte y gestores en el área de estudios culturales y de las comunicaciones. Trabajan con la metodología de creatividad compartida que determina el proceso de trabajo conjunto y los procesos de intercambio multidireccional, que cristalizan en un proyecto que se convierte en un "prototipo".

\subsection{MEDIALAB - MIT}

Su origen está en el Grupo de Máquinas de Arquitectura fundado en 1967 en el MIT. Es el centro donde se desarrollaron las primeras experiencias del diseño asistido por ordenador, Computer Aided Design (CAD). Este Laboratorio origina la expresión Medialab que se ha convertido en un nombre genérico en diferentes contextos.

En sus inicios el edificio "Centro de Arte y Tecnología de Medios", estaba destinado a la actividad entre arte, ciencia e ingeniería, que les permitía remodelar las tecnologías de comunicación. Es un espacio de vanguardia de las nuevas tecnologías y sus descubrimientos adquieren gran visibilidad. Una iniciativa realizada con aportes económicos de una empresa japonesa de tecnología, "Centro Okawa para el Futuro de la Infancia"; que investigó la influencia en los niños que viven, aprenden y juegan en la era digital. 


\section{METODOLOGÍAS ARTÍSTICAS DE INVESTIGACIÓN EN EDUCACIÓN}

Utilizan los conocimientos de actividades artísticas (danza, fotografía, música, teatro, video, etc.), para plantear problemas, obtener datos y resultados. Son una nueva forma investigación en ciencias humanas y sociales que trabajan la creación artística, fusionando la investigación científica y la innovación creativa.

Las Metodologías Artísticas de Investigación se inician en la investigación educativa y en la investigación en Educación Artística, utilizan las artes para hacer investigación. Sus conclusiones tienen multiplicidad de significados y existen varias metodologías artísticas como: Art For Scholarship's Sake, A/R/Tography, Arts-based Educational Research, Art-based Visual Research, Arts-based Research y Art-informed Research. Para la realización de los proyectos de los alumnos se explico y expuso la metodología A/R/Tography como una comunidad de artistas donde la figura del artista, investigador y profesor es un rol único y ofrece a través de la comunicación en el grupo múltiples posibilidades interdisciplinares.

A/R/Tography son los términos ingleses "Artist", "Researcher" y "Teacher", junto con "graphy" representación gráfica. Esta metodología ha evolucionado desde sus inicios en la Universidad de Columbia Británica en Vancouver hasta convertirse en una investigación compleja al implicar al conjunto de la persona, no sólo en su investigación, sino en aspectos de género, como docente, artista, etc.

Busca la fusión entre las actividades docentes, artísticas e investigadoras en un proyecto integrado, entre la enseñanza, la creación artística y la investigación científica (Irwin, 2004; Springgay, 2002).

\section{ARTE MULTIMEDIA}

El new media art son obras que utilizan la tecnología de los medios de comunicación emergentes e indagan las probabilidades estéticas, culturales y políticas; el arte digital, arte interactivo y multimedia. Incluyen manifestaciones artísticas: videoarte, multimedia, net.art, arte interactivo, fotomontaje digital, realidad virtual, inteligencia artificial, cine experimental, instalaciones, performances, etc. Internet es una herramienta clave para artistas del arte multimedia, así como el vídeo, videojuegos, telefonía inalámbrica y sistemas GPS. Los autores utilizan estas innovación para criticar o con un fin experimental y las transforman en proyectos artísticos.

\section{INSTALACIONES, INTERVENCIONES, PERFORMANCES Y HAPPENINGS EN PROYECTOS INTERDISCIPLINARES}

Las creaciones de artistas que han utilizado el cuerpo para sus trabajos demuestra la inclusión de ideas de distintas disciplinas. En 1910 y 1920, los dadaístas Tristan Tzara y Kurt Schwitters realizaron actuaciones irreverentes, interpretativas y multidisciplinares incorporando realidades corporales para desafiar la "pretenciosidad" de las representaciones artísticas tradicionales. Los dadaístas crearon arte en lugares que consideraban más reales e interesantes que los museos y salas de arte utilizando cafeterías, periódicos, calles, etc. El surrealismo evolucionó a partir del dadaísmo y añadió un aspecto psicológico y popularizó el interés freudiano por el sexo, el mundo de los sueños y el inconsciente, cuya máxima representación es Pietá or Revolution by Night (1923) de Max Ernst. El collage, fotomontaje, instalación, performance, los ambientes, los montajes dadaístas y surrealistas rompieron con el marco y el plano de la pintura y empezaron a formar parte de la vida cotidiana.

John Cage, Marcel Duchamp, Allan Kaprow y Kazuo Shiraga, utilizaron espacios alejados de las galerías para expresar sus ideas con obras multidisciplinares y performances basadas en el procedimiento. 


\section{CARTOGRAFIAS DE CREACIÓN}

La exposición presentada en el Caixa Forum Madrid titulada Cartografías contemporáneas dibujando el pensamiento ${ }^{1}$ presenta las cartografías creadas por artistas del s. XX y XXI que se interrogan sobre la representación. Cartografías de espacios mentales y físicos que crean reflexiones sobre los diversos tipos de espacios (virtuales, invisibles, utopías o heterotopías). Esto nos hace más conscientes de la idea de ilusión que tenemos de la realidad, de las dificultades que tenemos para representar el mundo, de las nociones de ideología y poder intrínsecos cuando se representa una realidad objetiva. Esta muestra es un ejemplo de cómo se puede presentar un espacio físico en una cartografía por diferentes artistas, a partir de ahí se creo el concepto Cartografías de Creación: visualizar en imagen los procesos artísticos que se pueden desarrollar en un proyecto interdisciplinar.

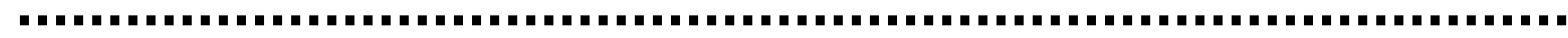

\section{PROYECTOS ARTÍSTICOS INTERDISCIPLINARES EN EL AULA}

Las instalaciones, intervenciones, performances y happenings se utilizan para introducir al alumno en el arte contemporáneo y en la realización de su práctica artística. La actividad se estructura a través de una síntesis de la obra con 300 palabras que explican el proyecto, la segunda parte es el proyecto artístico interdisciplinar en el se que deben mezclar 2 ó 3 disciplinas artísticas como: video, instalación, intervención, performance, happening, sonido, etc; con los conceptos teóricos vistos en el aula. Por ultimo se realiza una cartografía de creación, es decir se explica mediante un mapa visual el proceso artístico seguido para realizar el proyecto desde el inicio a su finalización.

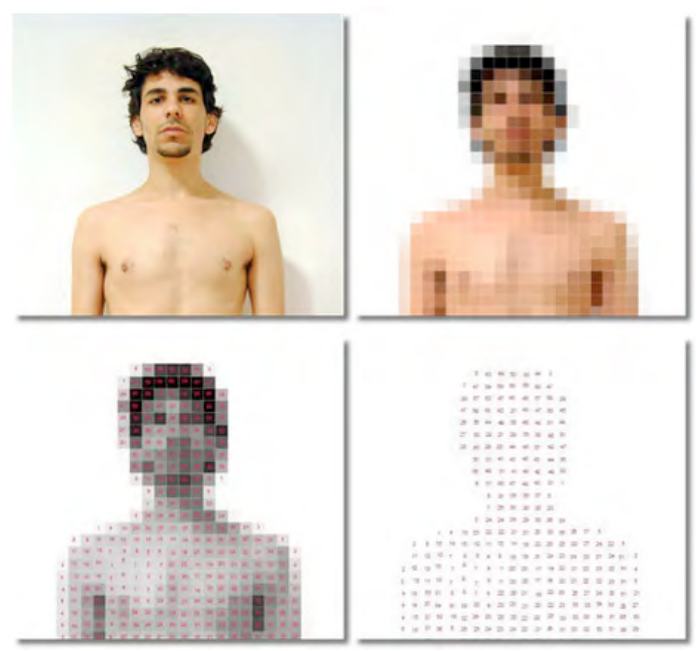

Fig. 1. "Pixelman", 2014. GONZÁLEZ RODRIGUEZ, M. PADILLA BARRERA, E. STICKLAND, F.

\footnotetext{
${ }^{1}$ Exposición CaixaForum Madrid. Cartografías contemporáneas. Dibujando el pensamiento. 21 Noviembre 2012 al 24 Febrero de 2013.
} 


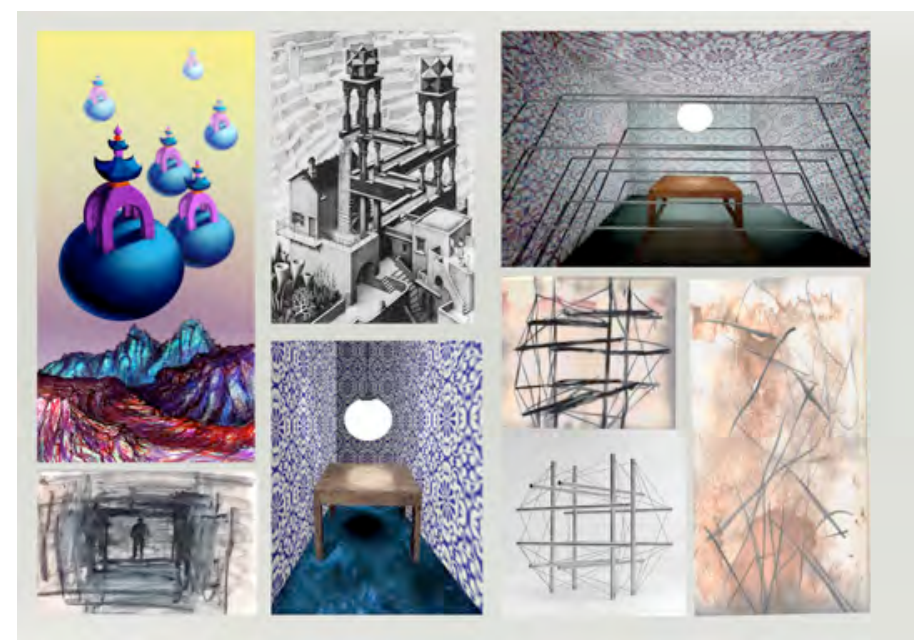

Fig. 2. “Cartografía de creación 3", 2014.

LEÓN CONCEPCIÓN, J. I. OJEDA ALONSO, A. L. RIVERO TRUJILLO, D.

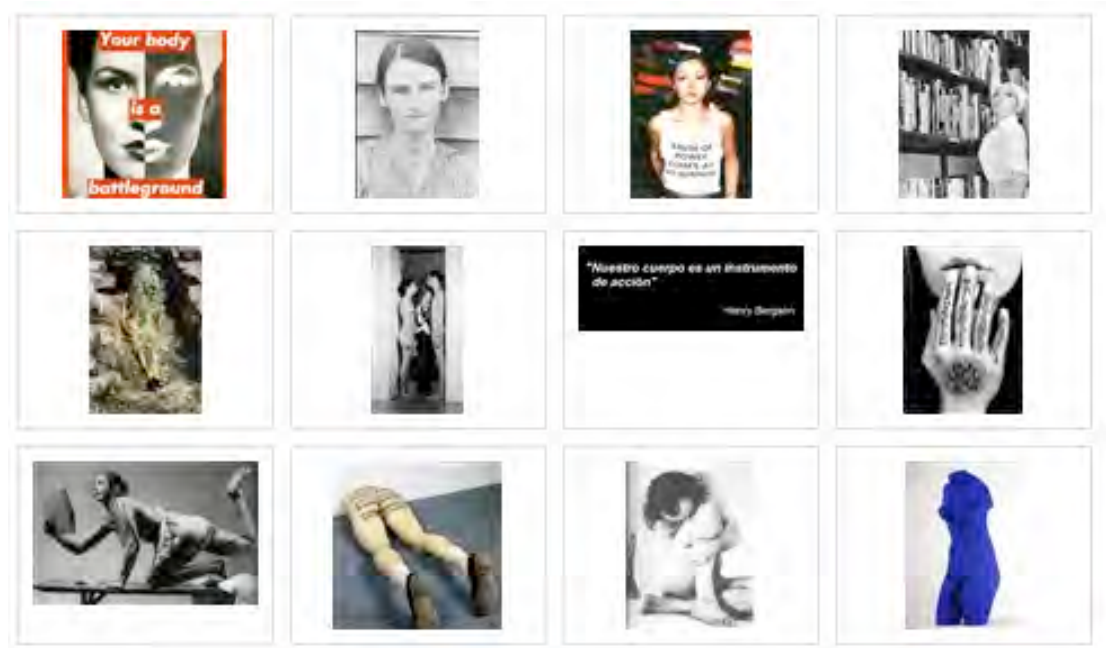

Fig. 3. "Cartografía de creación”, 2014.

LÓPEZ ARENCIBIA, V. MUÑOZ CANO, J. RESINA LÓPEZ, M. 


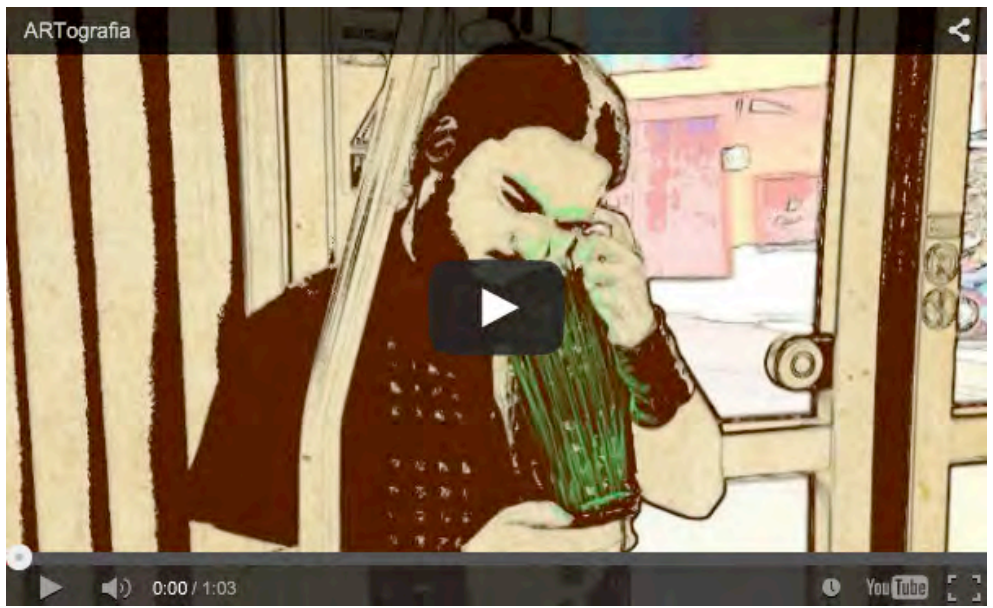

Fig. 4. "Proyecto artístico visual. Interdisciplinar", 2015.

SÁNCHEZ GRANDE IZQUIERDO, J. HERNANDEZ HERNANDEZ, S. MESA MENDEZ,

T. KOHLER, $S$.

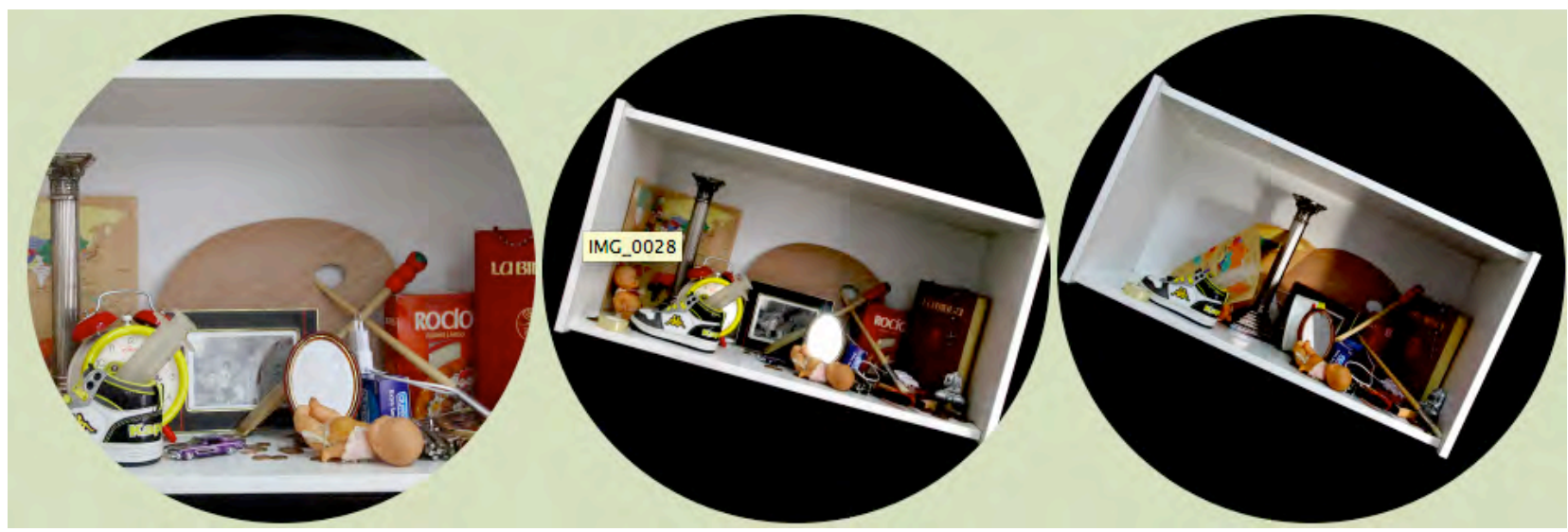

Fig. 5. "Proyecto artístico visual. Secuencia de rotación", 2015.

YANES ARMAS, D. CANO HUERTAS, S. ATECA MORENO, L. AMADOR GARCIA, E. M. ISAKOVIC, A.

\section{REFERENCIAS DOCUMENTALES}

AAVV. (2006). El cuerpo del artista. Nueva York: Phaidon Press.

AAVV. (2009). Art School (Propositions for the $21^{\text {st }}$ century). Massachusetts: The MIT Press.

CaixaForum Madrid. Exposición: “Cartografías contemporáneas. Dibujando el pensamiento". [En línea] <Disponible en web: http://obrasocial.lacaixa.es/nuestroscentros/caixaforummadrid/cartografiascontemporaneas_es.html > [Consulta: 3 de Mayo de 2015].

DÍAZ OBREGÓN, R. (2003). Arte contemporáneo y educación artística los valores potencialmente educativos de la instalación. Tesis doctoral. Madrid: Universidad Complutense de Madrid. 
MARÍN VIADEL, R. ROLDÁN, J. (2012). Metodologías artísticas de investigación en Educación. Las metodologías artísticas de Investigación y la Investigación educativa basada en las Artes Visuales. Capitulo 1. Málaga: Aljibe.

MARTINEZ PIMENTEL, L. C. (2008). “El cuerpo híbrido en la danza: transformaciones en el lenguaje coreográfico a partir de las tecnologías digitales. análisis teórico y propuestas experimentales." Tesis doctoral. Valencia: Universidad Politécnica de Valencia.

Official Website: John Cage. [En línea] <Disponible en Web: http://johncage.org/ > [Consulta: 22 de Abril de 2015].

Official Website: Black Mountain College. [En línea] <Disponible en web: http://www.blackmountaincollege.org/ > [Consulta: 22 de Abril de 2015].

Official Website: Merce Cunningham. [En línea] <Disponible en web: http://www.mercecunningham.org/newwebsite/> [Consulta: 22 de Abril de 2015].

PASTOR, R. (2011). Artes Plásticas y danza: Propuesta para una didáctica interdisciplinar. Tesis doctoral. Madrid: Universidad Complutense de Madrid.

SHANKEN, A. E. (2009). Art and Electronic Media. Nueva York: Phaidon Press.

SPRINGGAY, S. IRWIN, R. L. y KIND, S. (2005). A/R/Tography as living inquiry through art and text. [En línea] <Disponible en Web: http://qix.sagepub.com/content/11/6/897.abstract >. [Consulta: 10 de Mayo de 2015].

Tate Museum. Process art. Online Resources. Pagina Web oficial: Tate Museum [En línea] <Disponible en Web: http://www.tate.org.uk/learn/online-resources/glossary/p/process-art >. [Consulta: 13 de Abril de 2015].

The OpenEnded Group. [En línea] <Disponible en web: http://openendedgroup.com/artworks/loops.html > [Consulta: 22 de Abril de 2015].

The Solomon R. Guggenheim Foundation. Process art. U.S. and Europe, mid-1960s. Pagina Web oficial: Guggenheim Museum. [En línea] <Disponible en Web: http://www.guggenheim.org/new-york/collections/collectiononline/movements/195233 >. [Consulta: 13 de abril de 2015]

VILLAR ALÉ, R. E. (2013). “Procesos artísticos en laboratorios: ¿los medialabs un nuevo espacio para la educación artística?.” Tesis doctoral. Valladolid: Universidad de Valladolid.

PROYECTOS ALUMNOS. UNIVERSIDAD DE LA LAGUNA

GONZÁleZ RODRIGUEZ, M. PADILLA BARRERA, E. STICKLAND, F. "Proyecto A/r/tography" [En línea] <Disponible en web: http://wormlet77.wordpress.com/ > [Consulta: 10 de Mayo de 2015].

LEÓN CONCEPCIÓN, J. I. OJEDA ALONSO, A. L. RIVERO TRUJILLO, D. "Proyecto artístico visual. A/r/tography” [En línea] <Disponible en web: https://proyectoartography.wordpress.com/about/proyecto-artistico-visual_artography/ > [Consulta: 10 de Mayo de 2015].

LÓPEZ ARENCIBIA, V. MUÑOZ CANO, J. RESINA LÓPEZ, M. “A/r/tography. Arte y cuerpo” [En línea] <Disponible en web: http://artographycuerpo.blogspot.com.es/ > [Consulta: 10 de Mayo de 2015].

SÁNCHEZ GRANDE IZQUIERDO, J. HERNANDEZ HERNANDEZ, S. MESA MENDEZ, T. KOHLER, S. "Proyecto interdisciplinar" [En línea] <Disponible en web: https://aprendizajeyensenanza.wordpress.com/ > [Consulta: 10 de Mayo de 2015].

YANES ARMAS, D. CANO HUERTAS, S. ATECA MORENO, L. AMADOR GARCIA, E. M. ISAKOVIC, A. "Sobre la contingencia" [En línea] <Disponible en web: https://eduartproject.wordpress.com/ > [Consulta: 10 de Mayo de 2015]. 